\title{
PROXIMINALITY IN GENERALIZED DIRECT SUMS
}

\author{
DARAPANENI NARAYANA and T. S. S. R. K. RAO
}

Received 8 January 2004

\begin{abstract}
We consider proximinality and transitivity of proximinality for subspaces of finite codimension in generalized direct sums of Banach spaces. We give several examples of Banach spaces where proximinality is transitive among subspaces of finite codimension.
\end{abstract}

2000 Mathematics Subject Classification: 41A65, 41A50.

1. Introduction. Let $X$ be a Banach space and let $Y$ be a closed subspace of $X$. We recall that $Y$ is said to be a proximinal subspace of $X$ if for any $x \in X$ there exists a $y \in Y$ such that $d(x, Y)=\|x-y\|$.

In the first part of the paper, we study proximinal subspaces of finite codimension in generalized direct sums of Banach spaces (a concept due to Veselý [9], see below for the definition). Our motivation comes from some recent work of Indumathi [4] where she considered these questions for $c_{0}$-direct sums of a family of Banach spaces and proved the following.

THEOREM 1.1. Let $X=\left(\oplus X_{\lambda}\right)_{c_{0}}$ where each $X_{\lambda}$ is a Banach space for each $\lambda \in \Lambda$. Let $Y$ be a closed subspace of $X$ of finite codimension $n$ in $X$. Then $Y$ is proximinal in $X$ if and only if the following two conditions hold for every basis $\left\{f_{i}: 1 \leq i \leq n\right\}$ of $Y^{\perp}$, where $f_{i}=\left(f_{i, \lambda}\right)_{\lambda \in \Lambda}$ for $1 \leq i \leq n$ :

(i) for every $i, 1 \leq i \leq n, f_{i, \lambda}$ is nonzero only for finite number of indices $\lambda$,

(ii) $Y_{\lambda}=\cap_{i=1}^{n} \operatorname{ker} f_{i, \lambda}$ is proximinal in $X_{\lambda}$ for each $\lambda \in \Lambda$.

In the present paper, we prove an analogue of the above result for generalized direct sums. We next consider transitivity of proximinality among subspaces of finite codimension in $c_{0}$-direct sums. Here the motivation comes from [6] where transitivity was established among finite codimensional subspaces of $c_{0}$. We give several new examples of spaces where transitivity of proximinality holds among subspaces of finite codimension answering [3, Question 2] in the affirmative. We give a partial positive answer to this question in $c_{0}$-direct sums. For a Banach space $X$, let $N A(X)$ denote the set of norm attaining elements of $X^{*}$. We recall from [3] that $X$ is said to be an $R(1)$-space if $Y \subset X$ is of finite codimension and $Y^{\perp} \subset N A(X)$ implies $Y$ is proximinal. We show that this property is preserved by $c_{0}$-direct sums but not by $\ell_{1}$-direct sums. We only consider these topics for Banach spaces and leave the formulations in the more general setting of locally convex spaces open. An interested reader can consult the monograph [5] for a comprehensive treatment of proximinality in locally convex spaces. 
We now define generalized direct sums. Let $\Lambda$ be a nonempty set. Let $\left\{X_{\lambda}: \lambda \in \Lambda\right\}$ be a family of normed linear spaces. By $e_{\lambda}$, we denote the characteristic function of the singleton $\{\lambda\} \subset \Lambda$, that is, $e_{\lambda}\left(\lambda^{\prime}\right)=\delta_{\lambda \lambda^{\prime}}$.

Let $Y$ be a linear space, $\Lambda_{0} \subset \Lambda, y \in Y^{\Lambda}$. We denote by $\left.y\right|_{\Lambda_{0}}$, the element of $Y^{\Lambda}$ defined by

$$
\left.y\right|_{\Lambda_{0}}= \begin{cases}y(\lambda) & \lambda \in \Lambda_{0}, \\ 0 & \text { otherwise }\end{cases}
$$

Hence $\left.y\right|_{\Lambda_{0}}$ is the canonical projection of $y$ onto the subspace of functions whose support is contained in $\Lambda_{0}$ and $Y^{\Lambda_{0}}=\left\{y \in Y^{\Lambda} \mid \operatorname{supp}(y) \subset \Lambda_{0}\right\}$.

By a sequence space on $\Lambda$, we mean a normed linear space $(V, \gamma)$ such that $V$ is a linear subspace of $\mathbb{R}^{\Lambda}$.

DEFINITION 1.2. Let $(V, \gamma)$ be a sequence space on $\Lambda$ such that $\gamma$ is monotone on the nonnegative elements on $V$. Denote by $\left(\oplus X_{\lambda}\right)_{V}$ the linear space

$$
\left(\oplus X_{\lambda}\right)_{V}=\left\{x \in\left[\cup X_{\lambda}\right]^{\Lambda}: x(\lambda) \in X_{\lambda} \forall \lambda \in \Lambda,\|x(\cdot)\| \in V\right\}
$$

equipped with the norm $\|x\|_{V}=\gamma(\|x(\cdot)\|)$ where $\|x(\cdot)\|$ means the function $\lambda \rightarrow$ $\|x(\lambda)\|_{X_{\lambda}}$.

Let $\pi: \mathbb{R}^{\Lambda} \mapsto[0,+\infty]$ be a norm on $\mathbb{R}^{\Lambda}$ which is finite on the elements with finite support. By $S_{\pi}(\Lambda)$, we denote the linear space $S_{\pi}(\Lambda)=\left\{\xi \in \mathbb{R}^{\Lambda}: \pi(\xi)<+\infty\right\}$ equipped with the norm $\pi$.

DEFINITION 1.3. A norm $\pi: \mathbb{R}^{\Lambda} \mapsto[0,+\infty]$ will be called

(i) proper if it is finite on the elements with finite support,

(ii) finitely determined if for every $\xi \in \mathbb{R}^{\Lambda}$,

$$
\pi(\xi)=\sup \left\{\pi\left(\left.\xi\right|_{\Lambda_{0}}\right): \Lambda_{0} \text { is a finite subset of } \Lambda\right\},
$$

(iii) monotonic if $\pi(\xi) \leq \pi(\eta)$ whenever $|\xi| \leq|\eta| \xi, \eta \in \mathbb{R}^{\Lambda}$,

(iv) dual norm of a sequence space on $\Lambda$ if there exists $(V, \gamma)$ sequence space on $\Lambda$ (as defined above), containing basic vectors $e_{\lambda}$ as unit vectors and such that its dual $V^{*}$ is isometric with $S_{\pi}(\Lambda)$ and the isometric correspondence between $v^{*} \in V^{*}$ and $w \in S_{\pi}(\Lambda)$ is given by

$$
v^{*}(\xi)=\sum_{\lambda \in \Lambda} \xi(\lambda) w(\lambda) \quad \text { where } \xi \in V
$$

When $V=S_{\pi}(\Lambda)$ we will write $\left(\oplus X_{\lambda}\right)_{\pi}$ instead of $\left(\oplus X_{\lambda}\right)_{V}$.

EXAMPLE 1.4. Let $1 \leq p \leq+\infty$. Let $\pi: \mathbb{R}^{\Lambda} \mapsto[0,+\infty]$ be the classical $\ell^{p}$-norm. Then $\pi$ is monotonic, proper, and finitely determined, and we have $S_{\pi}(\Lambda)=\ell^{p}(\Lambda),\left(\oplus X_{\lambda}\right)_{\pi}=$ $\left(\oplus X_{\lambda}\right)_{\ell p}$. 
Each classical $\ell^{p}$-norm is a dual norm of a sequence space on $\Lambda$, with the predual $V$ given by

$$
V= \begin{cases}c_{0}(\Lambda) & \text { if } p=1 \\ \ell^{q}(\Lambda) & \text { if } 1<p<+\infty, \frac{1}{p}+\frac{1}{q}=1 \\ \ell^{1}(\Lambda) & \text { if } p=+\infty\end{cases}
$$

The following lemma was proved in [9].

LEMMA 1.5. Let $\pi: \mathbb{R}^{\Lambda} \mapsto[0,+\infty]$ be a norm which is monotonic, proper, and finitely determined. Let $X_{\lambda}$ be a Banach space for every $\lambda \in \Lambda$. Then

(a) $\left(\oplus X_{\lambda}\right)_{\pi}$ and $S_{\pi}(\Lambda)$ are Banach spaces,

(b) if $\pi$ is a dual norm of a sequence space on $\Lambda$, then the space $\left(\oplus X_{\lambda}^{*}\right)_{\pi}$ is isometric to a dual space.

2. Proximinality in generalized direct sums. We need the following theorems of Garkavi (see [7, pages 94-95]).

THEOREM 2.1. Let $Y$ be a closed subspace of finite codimension in a normed linear space $X$. Then $Y$ is proximinal if and only if for each $\Phi \in\left(Y^{\perp}\right)^{*}$, there exists $x \in X$ such that $\|\Phi\|=\|x\|$ and $\Phi(f)=f(x)$ for all $f \in Y^{\perp}$.

The following result is easy to deduce from the above theorem.

THEOREM 2.2. Let $X$ be a normed linear space and $Y$ a closed subspace of finite codimension in $X$. Then $Y$ is proximinal in $X$ if and only if every closed subspace $Z \supseteq Y$ of $X$ is proximinal in $X$.

As an immediate consequence one has that if a finite codimensional subspace $Y \subset X$ is proximinal, then $Y^{\perp} \subset N A(X)$.

We extend Indumathi's result on $c_{0}$-direct sums which is mentioned in the last section, to generalized direct sums. We now introduce the notations that we are going to use in this result.

Let $\left\{X_{\lambda}: \lambda \in \Lambda\right\}$ be a family of Banach spaces. Let $(V, \gamma)$ be a sequence space on $\Lambda$ and $\pi$ a dual norm of a sequence space on $\Lambda$ such that $X^{*}=\left(\oplus X_{\lambda}^{*}\right)_{\pi}$ where $X=\left(\oplus X_{\lambda}\right)_{V}$. We recall from Definition 1.3(iv) that this in particular means that $V$ has the canonical basis vectors as unit vectors. Let $Y \subset X$ be a closed subspace of finite codimension. Let $f_{i}=$ $\left(f_{i, \lambda}\right)_{\lambda \in \Lambda}$ where $i=1, \ldots, n$, be in $Y^{\perp}$ such that $Y=\cap_{i=1}^{n} \operatorname{ker} f_{i}$ and let $Z_{\lambda}=\cap_{i=1}^{n} \operatorname{ker} f_{i, \lambda}$. Assume that each $Z_{\lambda}$ is proximinal in corresponding $X_{\lambda}$. Now we have the following.

THEOREM 2.3. Every finite codimensional closed subspace $Y$ of the above form is proximinal in $X$ for every dual norm $\pi$ if and only if all but finitely many $X_{i}$ 's are $\{0\}$.

Proof. First suppose that all but finitely many $X_{\lambda}$ 's are trivial spaces. Let $Y \subset X$ be of finite codimension. By our assumption, $Z_{\lambda}$ is proximinal in respective $X_{\lambda}$. Since all but finitely many $X_{\lambda}$ 's are trivial, $f_{i}$ 's have only finitely many nonzero terms. Consider $A=\cup_{i=1}^{n}\left\{\lambda \in \Lambda: f_{i, \lambda} \neq 0\right\}$. We have that $|A|<\infty$. 
Let $G$ be a subspace of $X$ such that $G=\left\{x_{\left.\right|_{A}}: x \in X\right\}$ and $Z_{\lambda}=\cap_{i=1}^{n} \operatorname{ker} f_{i \lambda}$ for $\lambda \in A$. For $\lambda \in A$, each $Z_{\lambda}$ is a proximinal subspace of finite codimension in $X_{\lambda}$. Further if $Z=\left(\oplus Z_{\lambda}\right)_{V}$, then we show that $Z$ is a proximinal subspace of finite codimension in $G$. Let $g=\left(g_{\lambda}\right)$ be in $G$. For every $g_{\lambda}$ we have $z_{\lambda}^{0}$ in $Z_{\lambda}$ such that $\left\|g_{\lambda}-z_{\lambda}^{0}\right\|=d\left(g_{\lambda}, Z_{\lambda}\right)$. Let $z^{0}=\left(z_{\lambda}^{0}\right) \in Z$. Now $\left\|g-z^{0}\right\|_{V}=\gamma\left(\left\|g_{\lambda}-z_{\lambda}^{0}\right\|\right) \leq \gamma\left(\left\|g_{\lambda}-z_{\lambda}\right\|\right)$ for $z=\left(z_{\lambda}\right) \in Z$, by monotonicity of $\gamma$. Thus $Z$ is proximinal in $G$. Set $Y_{0}=\cap_{i=1}^{n}\left\{x_{\left.\right|_{A}}: x=\left(x_{\lambda}\right) \in X\right.$ and $\left.\sum_{\lambda \in A} f_{i \lambda}\left(x_{\lambda}\right)=0\right\}$. Then $Y_{0}$ is a subspace of $G, Z \subset Y_{0} \subset G$. Now by Theorem 2.2, we conclude that $Y_{0}$ is proximinal in $G$. Again using the monotonicity of the norm $\gamma$ it can be easily seen that this implies that $Y$ is proximinal in $X$.

Conversely we assume that every finite codimensional closed subspace $Y \subset X$ of the form considered earlier is proximinal in $X$. Suppose infinitely many $X_{\lambda}$ 's are nontrivial. Then as in [4], we give an example of a subspace $Y$ of $X$ of codimension 2 such that $Y$ is not proximinal in $X$.

CONSTRUCTION OF THE EXAMPLE. Let $\gamma$ be a norm which is not equal to the $c_{0}$ norm. In particular we take $\gamma=\ell_{1}$. Assume without loss of generality that $\Lambda=\mathbb{N}$ and $X=\ell_{1}$. Then $X^{*}=\ell_{\infty}$. Take $f_{1}=(1,0,3 / 4,4 / 5, \ldots, n /(n+1), \ldots)$ and $f_{2}=(0,1,3 / 4,4 / 5, \ldots$, $n /(n+1), \ldots)$ in $N A(X)=\left\{\left(\alpha_{n}\right) \in \ell_{\infty}\right.$ : there exists $n_{0} \in \mathbb{N}$ such that $\left.\left\|\left(\alpha_{n}\right)\right\|_{\infty}=\left|\alpha_{n_{0}}\right|\right\}$. Let $x=(1,1,0,0, \ldots)$. Then $f_{1}(x)=1$ and $f_{2}(x)=1$.

Since $f_{1}$ and $f_{2}$ are in $N A(X)$, $\operatorname{ker} f_{1}$ and $\operatorname{ker} f_{2}$ are proximinal hyperplanes of $X$. Let $Y=\cap_{i=1}^{2} \operatorname{ker} f_{i}$ so that $\operatorname{dim}(X / Y)=2$. We show that $Y$ is not proximinal in $X$. Clearly $d(x, Y)=\left\|\left.x\right|_{Y^{\perp}}\right\| \geq 1$.

We now claim that $d(x, Y)=1$. Select $x_{n}$ in $\mathbb{R}$ such that $x_{1}=1=x_{2}$ and for $n \geq 3$, put $x_{n}=-(n+1) / n$. Define $y_{k}$ in $X$ for $k \geq 3$ by

$$
y_{k}(n)= \begin{cases}x_{n} & \text { if } n \in\{1,2, k\} \\ 0 & \text { otherwise }\end{cases}
$$

Then $f_{i}\left(y_{k}\right)=0$ for $i=1,2$ and so $y_{k} \in Y$ for all $k$. Further $\left\|x-y_{k}\right\|=(k+1) / k \rightarrow 1$ as $k \rightarrow \infty$. Hence $d(x, Y)=1$. Now if there is a $y_{0} \in Y$ such that $\left\|x-y_{0}\right\|=d(x, Y)=1$, then $f_{1}\left(x-y_{0}\right)=1=\left\|x-y_{0}\right\|$ and $f_{2}\left(x-y_{0}\right)=1=\left\|x-y_{0}\right\|$. Since the first equality implies $y_{0}=e_{1}$, clearly the second equality cannot hold. Thus $Y$ is not proximinal.

We next show that any proximinal subspace of finite codimension in $X=\left(\oplus X_{\lambda}\right)_{c_{0}}$ is also a proximinal subspace of $W=\left(\oplus X_{\lambda}\right) \ell_{\infty}$. The proof uses ideas similar to the ones given above and the well-known facts, $X$ is a proximinal subspace of $W$ (this can be seen by verifying the "3-ball property" and concluding proximinality as in [2, Proposition II.1.1]) and that if $Z_{1}$ and $Z_{2}$ are two proximinal subspaces in $X_{1}$ and $X_{2}$, then $Z_{1} \oplus_{\ell_{\infty}} Z_{2}$ is proximinal in $X_{1} \oplus_{\ell_{\infty}} X_{2}$.

Corollary 2.4. Let $X=\left(\oplus X_{\lambda}\right)_{c_{0}}$ and let $W=\left(\oplus X_{\lambda}\right)_{\ell_{\infty}}$. Let $Y$ be a proximinal subspace of finite codimension in $X$. Then $Y$ is proximinal in $W$.

Proof. We follow the notations used during the proof of the above theorem. Let $X_{1}=\left(\oplus X_{\lambda, \lambda \in A}\right)_{\ell_{\infty}}$ and let $X_{2}=\left(\oplus X_{\lambda, \lambda \notin A}\right)_{c_{0}}$. Then one can see as in the above proof $Y_{0}$ 
is proximinal in $X_{1}$. Also as remarked above, $X_{2}$ is proximinal in $\left(\oplus X_{\lambda \notin A}\right)_{\ell_{\infty}}$. It is easy to see that $Y=Y_{0} \oplus_{\ell_{\infty}} X_{2}$. Therefore $Y$ is proximinal in $X_{1} \oplus_{\ell_{\infty}}\left(\oplus X_{\lambda \notin A}\right)_{\ell_{\infty}}=W$.

REMARK 2.5. Let $Y$ be a proximinal subspace of finite codimension in $X=\left(\oplus X_{\lambda}\right)_{c_{0}}$. Let $Y_{1}$ be the finite codimensional subspace in $W=\left(\oplus X_{\lambda}\right)_{\ell_{\infty}}$ obtained by intersecting the kernals of the same functionals that determine $Y$. Then by Theorem 2.3 we have that $Y_{1}$ is a proximinal subspace of $W$. We also get from the above corollary that $Y$ is proximinal in $Y_{1}$.

Our next result substantially improves on the above corollary when the component spaces are scalars. We retain the notations used above.

Proposition 2.6. Let $Y \subset \ell_{\infty}(I)$ be a subspace of finite codimension determined by finitely supported functionals in $\ell_{1}(I)$. Then $Y$ is proximinal, under the canonical embedding in $\ell_{\infty}^{* *}(I)$.

Proof. We have that $Y$ is a weak*-closed subspace and hence proximinal in $\ell_{\infty}(I)$. Let $X_{1}=\left(\oplus \mathbb{R}_{\lambda, \lambda \in A}\right)_{\ell_{\infty}}$ and $X_{2}=\left(\oplus \mathbb{R}_{\lambda, \lambda \notin A}\right)_{\ell_{\infty}}$. We have as before $Y=Y_{0} \oplus_{\ell_{\infty}} X_{2}$ and $\ell_{\infty}(I)=X_{1} \oplus_{\ell_{\infty}} X_{2}$. We next recall the well-known fact that any space of continuous functions on a compact set is proximinal in its bidual, see [8]. Thus $X_{2}$ which can be identified as the space of continuous functions on the Stone-Cech compactification of the index set, is proximinal in its bidual. Also $X_{1}$ is a finite-dimensional space. Therefore $Y$ is proximinal in $X_{1} \oplus_{\ell_{\infty}} X_{2}^{* *}=\ell_{\infty}^{* *}(I)$.

We now consider transitivity of proximinality among subspaces of finite codimension. We use the notation $Y \stackrel{p}{\subset} X$ to indicate that $Y$ is a proximinal subspace of $X$.

Definition 2.7. A Banach space $X$ is said to be a $P$-space (Pollul space) if proximinality is transitive for subspaces of finite codimension, that is, $Y \stackrel{p}{\subset} Z \stackrel{p}{\subset} \subset$, and the fact that both $Y$ and $Z$ are of finite codimension implies $Y \stackrel{p}{\subset} X$.

Well-known examples of $P$-spaces are $c_{0}$ space, reflexive spaces. Also the space of compact operators $\mathscr{K}\left(\ell_{2}\right)$ on the Hilbert space $\ell_{2}$ is a $P$-space. To see this, we note that we have from [1, Lemma 4.2] that $N A\left(\mathscr{K}\left(\ell_{2}\right)\right)$ is a linear space. Also from [1, Theorem 5.3] we know that $\mathscr{K}\left(\ell_{2}\right)$ is an $R(1)$-space. It thus follows from [3, Corollary 5] that $\mathscr{K}\left(\ell_{2}\right)$ is a $P$-space. This answers [3, Question 2]. See [3] for more general results on transitivity.

The following lemma gives a way of giving more examples of $P$-spaces.

LEMMA 2.8. Let $X$ be a $P$-space and let $Y \subset X$ be a proximinal subspace of finite codimension. Then $Y$ is a $P$-space.

Proof. Let $Z_{1} \stackrel{p}{\subset} Z_{2} \stackrel{p}{\subset} Y \stackrel{p}{\subset} X$, where both $Z_{1}$ and $Z_{2}$ are finite codimensional subspaces of $Y$. Since $X$ is a $P$-space, $Z_{2}$ is proximinal in $X$. Using the same reasoning this time with $Z_{2}$ and $X$, we see that $Z_{1}$ is proximinal in $X$ and hence in $Y$. Thus $Y$ is a $P$-space.

To motivate the results that we will be proving next we give the details of transitivity of proximinality for $c_{0}$. The proof we present here is simpler than the one in $[3,6]$. 
LEMMA 2.9. Let $X=c_{0}=(\oplus \mathbb{R})_{c_{0}}$. Let $Z \stackrel{p}{\subset} Y \stackrel{p}{\subset} X$ and $\operatorname{dim}(X / Y)=n<\infty, \operatorname{dim}(Y / Z)=$ $m<\infty$. Then $Z \stackrel{p}{\subset} X$.

Proof. We will give the proof when $n=1$ and given the nature of proximinal subspaces of finite codimension, the arguments are similar when $n>1$. Let $f \in N A(X)$ be such that $Y=\operatorname{ker} f$. Clearly $f$ has only finitely many nonzero terms.

Let $Z \stackrel{p}{\subset} Y, \operatorname{dim}(Y / Z)=m$. Let $f_{1}, f_{2}, \ldots, f_{m} \in Z^{\perp} \subset Y^{*}$ be such that

$$
Z=\left\{y \in Y: f_{i}(y)=0 \forall i, 1 \leq i \leq m\right\}
$$

Here $f_{1}, \ldots, f_{m} \in N A(Y)$. Let $\tilde{f}_{1}, \ldots, \tilde{f}_{m}$ be norm-preserving extensions of $f_{1}, \ldots, f_{m}$ onto $X$. Then

$$
Z=\left\{x \in X: f(x)=0, \tilde{f}_{i}(x)=0 \forall i, 1 \leq i \leq m\right\}
$$

As $f_{1}, \ldots, f_{m}$ are norm-attaining functionals on $Y, \tilde{f}_{1}, \ldots, \tilde{f}_{m}$ are also norm attaining on $X$, which implies $Z \stackrel{p}{\subset} X$.

The following corollary can also be deduced from some of our later results. However we prefer to present a proof here using above ideas.

Corollary 2.10. Let $X=\left(\oplus X_{\lambda}\right)_{c_{0}}$, where each $X_{\lambda}$ is a reflexive Banach space. Then $X$ is a $P$-space. Any proximinal subspace of finite codimension in $X$ is also a $P$-space.

Proof. We suppose that $Y$ and $Z$ are closed subspaces of $X$ such that $Z \stackrel{p}{\subset} Y \stackrel{p}{\subset} X$ with $\operatorname{dim}(X / Y)=n<\infty$ and $\operatorname{dim}(Y / Z)=m<\infty$. Then as before we can write $Y$ and $Z$ as $Y=\cap_{i=1}^{n} \operatorname{ker} f_{i}$ and $Z=\cap_{i=1}^{n+m} \operatorname{ker} f_{i}$ where $f_{1}, f_{2}, \ldots, f_{n+m} \in X^{*}$. Let $Z_{\lambda}=\cap_{i=1}^{n+m} \operatorname{ker} f_{i, \lambda} \subset$ $Y_{\lambda}=\cap_{i=1}^{n} \operatorname{ker} f_{i, \lambda} \subset X_{\lambda}$. Then $Z_{\lambda}$ is proximinal in $Y_{\lambda}$ as well as in $X_{\lambda}$ by reflexivity. So by Theorem 1.1, $Z$ is proximinal in $X$ which completes the proof. The second assertion follows from Lemma 2.8.

As seen in the above proof in the general case the main difficulty is to prove the proximinality of $Z_{\lambda}$ in $X_{\lambda}$. We now give a positive result for the validity of transitivity in the case of $c_{0}$-direct sums. To state the result we need the following notation.

Let $X=\left(\oplus X_{\lambda}\right)_{c_{0}}$, where $X_{\lambda}$ is a Banach space for each $\lambda \in \Lambda$. Let $Y$ and $Z$ be closed subspaces of $X$ such that $Z \subset Y \subset X$ with finite codimensions, that is, $\operatorname{dim}(X / Y)=n<$ $\infty$ and $\operatorname{dim}(Y / Z)=m<\infty$. Then we can write $Z$ as $Z=\cap_{i=1}^{n+m} \operatorname{ker} f_{i}$, where $f_{i}=\left(f_{i, \lambda}\right) \in$ $Z^{\perp} \subset X^{*}$ for $1 \leq i \leq n+m$. Let $Z_{\lambda}=\cap_{i=1}^{n+m} \operatorname{ker} f_{i, \lambda}$ for $\lambda \in \Lambda$. Assume that if $z=\left(z_{\lambda}\right) \in Z$, then $z_{\lambda} \in Z_{\lambda}$ for every $\lambda \in \Lambda$. Then we have the following.

Proposition 2.11. Suppose each $X_{\lambda}$ in the above direct sum is a P-space. With the above assumption on $Z$, if $Z \stackrel{p}{\subset} Y \stackrel{p}{\subset} X$, then $Z \stackrel{p}{\subset} X$.

Proof. We suppose that $Z \stackrel{p}{\subset} Y \stackrel{p}{\subset} X=\left(\oplus X_{\lambda}\right)_{c_{0}}$. Since $Z \stackrel{p}{\subset} Y$ and $Y \stackrel{p}{\subset} X$, there exists $f_{i} \in N A(X)$ for $1 \leq i \leq n+m$ such that $Y=\cap_{i=1}^{n} \operatorname{ker} f_{i}$ and $Z=\cap_{i=1}^{n+m} \operatorname{ker} f_{i}$ in $X$. Let $Z_{\lambda}=\cap_{i=1}^{n+m} \operatorname{ker} f_{i, \lambda} \subset \cap_{i=1}^{n} \operatorname{ker} f_{i, \lambda} \subset X_{\lambda}$. 
We claim that $Z_{\lambda}$ is proximinal in $Y_{\lambda}=\cap_{i=1}^{n} \operatorname{ker} f_{i, \lambda}$. Indeed let $y_{\lambda} \in Y_{\lambda}$. Now consider $\bar{y}=\left(0, \ldots, 0, y_{\lambda}, 0, \ldots\right)$. Then there exists $z^{0}=\left(z_{\lambda}^{0}\right)_{\lambda \in \Lambda} \in Z$ such that $d(\bar{y}, Z)=\left\|z^{0}-\bar{y}\right\|$. By our assumption $z_{\lambda}^{0} \in Z_{\lambda}$. We next show that $z_{\lambda}^{0}$ is a best approximation.

Let $z_{\lambda} \in Z_{\lambda}$ and consider $z=\left(0, \ldots, 0, z_{\lambda}, 0, \ldots\right), z \in Z$. Now $\left\|y_{\lambda}-z_{\lambda}\right\|=\|\bar{y}-z\| \geq$ $\left\|\bar{y}-z^{0}\right\| \geq\left\|y_{\lambda}-z_{\lambda}^{0}\right\|$ which implies that $z_{\lambda}^{0}$ is a best approximation.

Therefore $Z_{\lambda}$ is proximinal in $Y_{\lambda}$ for all $\lambda \in \Lambda$. Thus $Z_{\lambda}$ is proximinal in $X_{\lambda}$ by the transitivity property of $X_{\lambda}$. By Theorem $1.1, Z$ is proximinal in $X$.

Conversely we have the following.

Proposition 2.12. Let $X=\left(\oplus X_{\lambda}\right)_{V}$ be a $P$-space. Then $X_{\lambda}$ is a $P$-space for each $\lambda \in \Lambda$.

Proof. Fix $\lambda_{0} \in \Lambda$ and let $Z_{\lambda_{0}}$ and $Y_{\lambda_{0}}$ be closed subspaces of $X_{\lambda_{0}}$ such that $Z_{\lambda_{0}} \stackrel{p}{\subset}$ $Y_{\lambda_{0}} \stackrel{p}{\subset} X_{\lambda_{0}}$ with $\operatorname{dim}\left(X_{\lambda_{0}} / Y_{\lambda_{0}}\right)=n<+\infty$ and $\operatorname{dim}\left(Y_{\lambda_{0}} / Z_{\lambda_{0}}\right)=m<+\infty$. Now consider $Y=\left(\oplus Y_{\lambda}\right)_{V}$ and $Z=\left(\oplus Z_{\lambda}\right)_{V}$ where

$$
Y_{\lambda}=\left\{\begin{array}{ll}
X_{\lambda} & \text { if } \lambda \neq \lambda_{0}, \\
Y_{\lambda_{0}} & \text { if } \lambda=\lambda_{0},
\end{array} \quad Z_{\lambda}= \begin{cases}X_{\lambda} & \text { if } \lambda \neq \lambda_{0} \\
Z_{\lambda_{0}} & \text { if } \lambda=\lambda_{0} .\end{cases}\right.
$$

Clearly $Z$ and $Y$ are subspaces of finite codimension in $X$. It is easy to see that $Z \stackrel{p}{\subset}$ $Y \stackrel{p}{\subset} X$. Therefore $Z \stackrel{p}{\subset} X$ since $X$ is a $P$-space.

Now we claim that $Z_{\lambda_{0}} \stackrel{p}{\subset} X_{\lambda_{0}}$. Let $x_{\lambda_{0}} \in X_{\lambda_{0}}$. Consider $x_{0}=\left(0, \ldots, x_{\lambda_{0}}, 0, \ldots\right) \in X$. Then there exists $Z_{0}=\left(z_{\lambda}^{0}\right) \in Z$ such that $\left\|x_{0}-z_{0}\right\|=d\left(x_{0}, Z\right)$. Now we show that $z_{\lambda_{0}}^{0}$ is a best approximation of $x_{\lambda_{0}}^{0}$ from $Z_{\lambda_{0}}$. Consider $z=\left(0, \ldots, 0, z_{\lambda_{0}}, 0, \ldots\right)$ where $z_{\lambda_{0}} \in Z_{\lambda_{0}}$. Clearly $z \in Z$. Now $\left\|x_{\lambda_{0}}-z_{\lambda_{0}}\right\|=\left\|x_{0}-z\right\| \geq\left\|x_{\lambda_{0}}-z_{\lambda_{0}}^{0}\right\|$ which implies that $z_{\lambda_{0}}^{0}$ is a best approximation of $x_{\lambda_{0}}$ from $Z_{\lambda_{0}}$. Thus $Z_{\lambda_{0}} \stackrel{p}{\subset} X_{\lambda_{0}}$. Hence $X_{\lambda_{0}}$ is a $P$-space. Since $\lambda_{0}$ is arbitrary, the conclusion follows.

REMARK 2.13. Identifying $\mathscr{K}\left(c_{0}\right)$ as $\left(\oplus \ell_{1}\right)_{c_{0}}$ we see that as $\ell_{1}$ is not a $P$-space (see [3, page 137]), $\mathscr{K}\left(c_{0}\right)$ is not a $P$-space.

We now prove that being $R(1)$ is invariant under $c_{0}$-direct sums. Using the same arguments presented in the converse part of the following proposition, one can show that this part holds for generalized direct sums also.

Proposition 2.14. Let $X=\left(\oplus_{\lambda} X_{\lambda}\right)_{c_{0}}$. Then $X$ is an $R(1)$-space if and only if each $X_{\lambda}$ is an $R(1)$-space.

Proof. Let $Y \subset X$ be a closed subspace of finite codimension and $Y^{\perp} \subset N A(X)$. Then there exists $f_{1}, \ldots, f_{n} \in X^{*}$ such that $Y=\cap_{i=1}^{n} \operatorname{ker} f_{i}$.

As $f_{i} \in N A(X)$, only finitely many coordinates of $f_{i}$ 's are nonzero for each $i$. Thus $f_{i}=\left(f_{i, \lambda}\right)_{\lambda \in \Lambda}, f_{i, \lambda} \neq 0$ for finitely many $\lambda \in \Lambda$.

It is easy to see that if a functional in $X^{*}$ with only finitely many nonzero coordinates attains its norm, then each nonzero component functional attains its norm. Thus since $Y^{\perp} \subset N A(X)$, following the notation of Theorem 1.1 we see that for any $\lambda, Y_{\lambda}^{\perp} \subset N A\left(X_{\lambda}\right)$. We thus have $Y_{\lambda}$ is proximinal in $X_{\lambda}$ since each $X_{\lambda}$ is an $R(1)$-space. Now by Theorem 1.1, $Y$ is proximinal which implies that $X$ is an $R(1)$-space. 
To see the converse it is enough to consider the case $X=X_{1} \oplus_{\infty} X_{2}$ where $X$ is an $R$ (1)-space. Now let $Y \subset X_{1}$ be such that $Y^{\perp} \subset N A\left(X_{1}\right)$ and $Y^{\perp}=\operatorname{span}\left\{f_{1}, \ldots, f_{k}\right\}$. It is easy to see that $Y^{\prime}=\operatorname{span}\left\{\left(f_{1}, 0\right), \ldots,\left(f_{k}, 0\right)\right\} \subset N A(X)$. Thus the preannihilator of $Y^{\prime}$ is a proximinal subspace of $X$. Hence $Y$ is proximinal in $X_{1}$. Therefore $X_{1}$ is an $R(1)$-space.

The following corollary allows us to give more examples of $P$-spaces. In particular it shows that $\left(\oplus \mathscr{K}\left(\ell_{2}\right)\right)_{c_{0}}$ is a $P$-space. As mentioned before it also gives another proof of Corollary 2.10. We omit its easy proof.

Corollary 2.15. Let $X=\left(\oplus X_{\lambda}\right)_{c_{0}}$, where each $X_{\lambda}$ is an $R(1)$-space and $N A\left(X_{\lambda}\right)$ is a linear space. Then $X$ has the same properties. In particular $X$ is a $P$-space.

REMARK 2.16. Proposition 2.14 does not hold for generalized direct sums. It is not true even for $\ell_{1}$-direct sums over infinite index. The same example presented in the proof of Theorem 2.3 works in this case also.

ACKNOWLEDGMENT. The first author thanks the Indian Statistical Institute, Bangalore, for its hospitality during his stay in July-August 2002. The second author's research was partially supported by an Indo-French project, IFCPAR Grant 2601-1.

\section{REFERENCES}

[1] G. Godefroy, V. Indumathi, and F. Lust-Piquard, Strong subdifferentiability of convex functionals and proximinality, J. Approx. Theory 116 (2002), no. 2, 397-415.

[2] P. Harmand, D. Werner, and W. Werner, M-Ideals in Banach Spaces and Banach Algebras, Lecture Notes in Mathematics, vol. 1547, Springer-Verlag, Berlin, 1993.

[3] V. Indumathi, On transitivity of proximinality, J. Approx. Theory 49 (1987), no. 2, 130-143.

[4] _ Proximinal subspaces of finite codimension in direct sum spaces, Proc. Indian Acad. Sci. Math. Sci. 111 (2001), no. 2, 229-239.

[5] G. Isac and V. Postolică, The Best Approximation and Optimization in Locally Convex Spaces, Approximation \& Optimization, vol. 2, Peter Lang, Frankfurt am Main, 1993.

[6] W. Pollul, Reflexivität und Existenz-Teilräume in der linearen Approximationstheorie, Gesellschaft für Mathematik und Datenverarbeitung, Bonn, 1972.

[7] I. Singer, Best Approximation in Normed Linear Spaces by Elements of Linear Subspaces, Grundlehren der Mathematischen Wissenschaften in Einzeldarst, Band 171, SpringerVerlag, Berlin, 1970.

[8] _ The Theory of Best Approximation and Functional Analysis, Conference Board of the Mathematical Sciences Regional Conference Series in Applied Mathematics, vol. 13, Society for Industrial and Applied Mathematics, Pennsylvania, 1974.

[9] L. Veselý, Generalized centers of finite sets in Banach spaces, Acta Math. Univ. Comenian. (N.S.) 66 (1997), no. 1, 83-115.

Darapaneni Narayana: Equipe d'Analyse, Université Paris 6, 4 Place Jussieu, 75252 Paris Cedex 05, France

Current address: Department of Mathematics, Indian Institute of Sciences, Bangalore 560012, India

E-mail address: narayana@math.i isc.ernet.in

T. S. S. R. K. Rao: Statistics and Mathematics Unit, Indian Statistical Institute, Bangalore 560059, India

E-mail address: tss@isibang.ac.in 


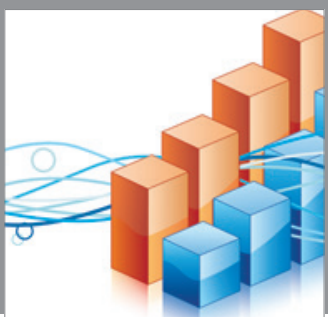

Advances in

Operations Research

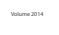

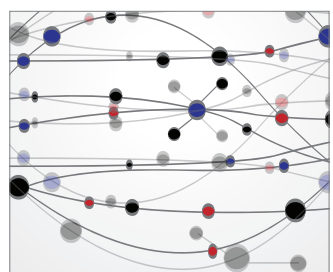

\section{The Scientific} World Journal
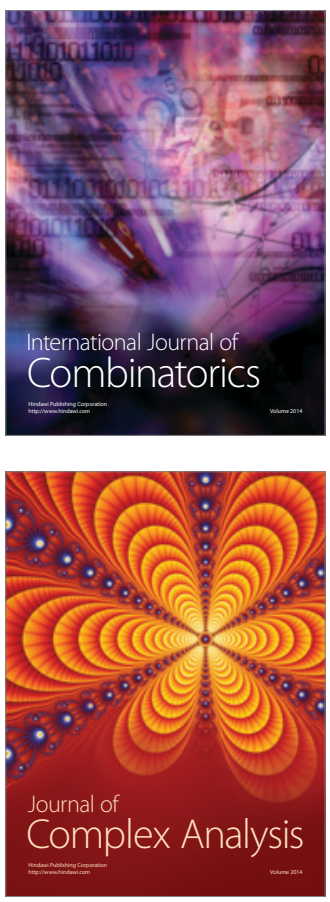

International Journal of

Mathematics and

Mathematical

Sciences
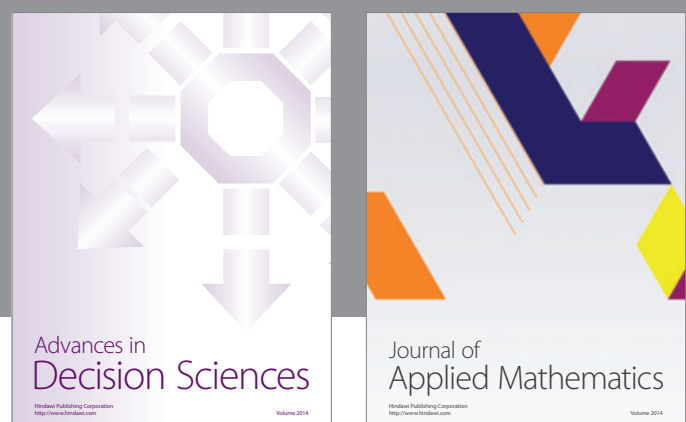

Journal of

Applied Mathematics
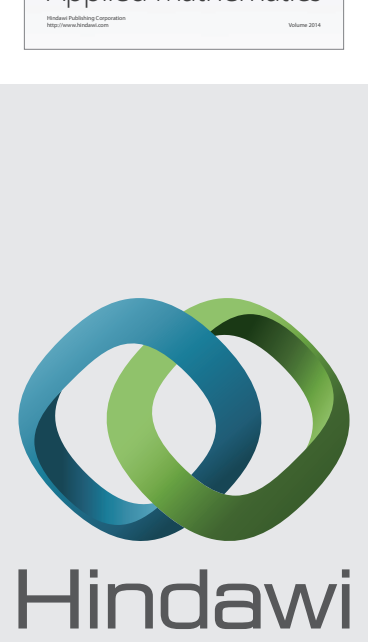

Submit your manuscripts at http://www.hindawi.com
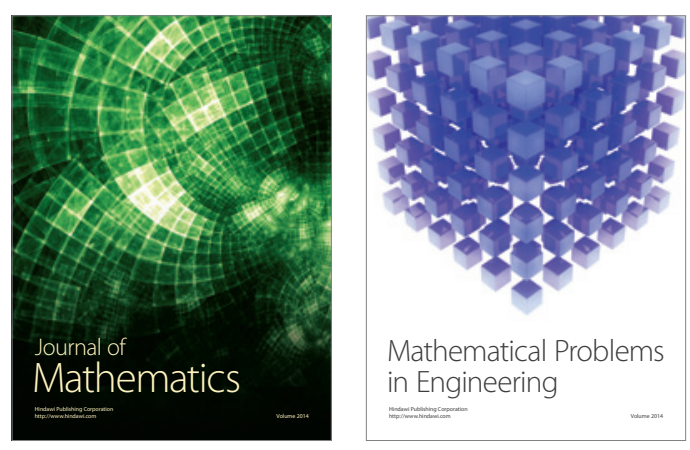

Mathematical Problems in Engineering
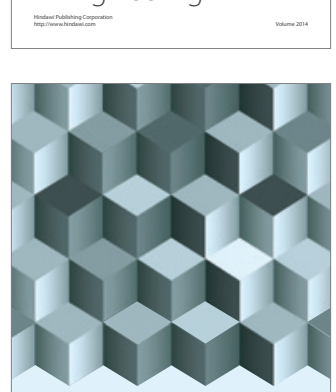

Journal of

Function Spaces
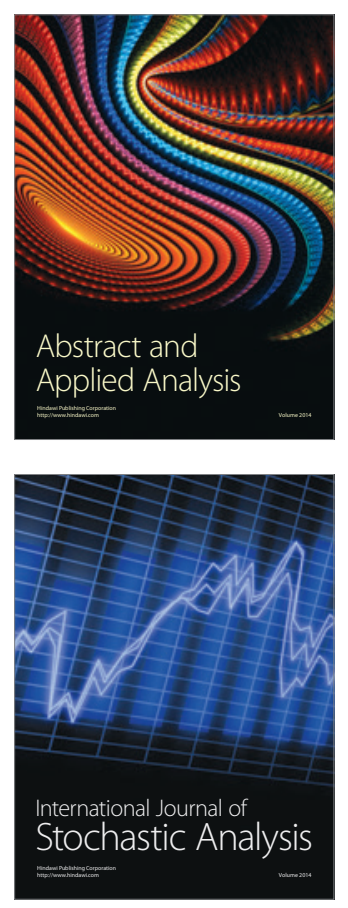

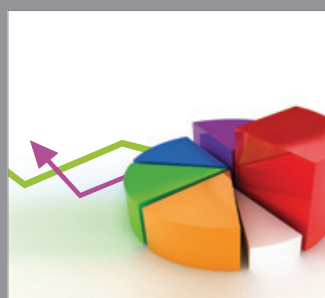

ournal of

Probability and Statistics

Promensencen
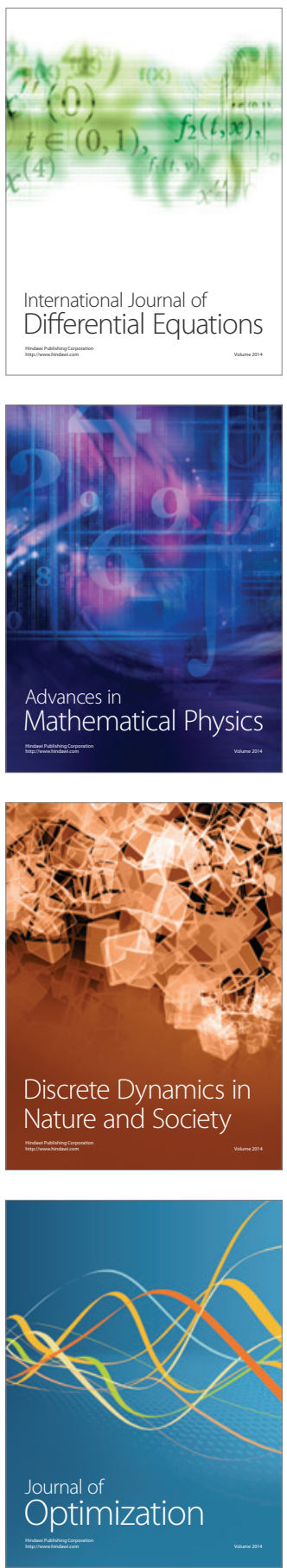